\title{
Negative Regulation of Inflammatory Response Process
}

National Cancer Institute

\section{Source}

National Cancer Institute. Negative Regulation of Inflammatory Response Process. NCI

Thesaurus. Code C40857.

An Antiinflammatory Effect involves interference with, or restraint of, local events that constitute part of innate immunity, involve increased blood supply, and infiltration and activity of leukocytes at a site of injury or disease. Inflammation is induced by release of vasodilatory, opsonizing, and chemotactic mediators that attract phagocytizing macrophages and leukocytes; dilate local blood vessels, increasing local blood flow and capillary permeability; and induce pain. 